\title{
Segmental, Synaptic Actions of Commissural Interneurons in the Mouse Spinal Cord
}

\author{
Katharina A. Quinlan and Ole Kiehn \\ Mammalian Locomotor Laboratory, Department of Neuroscience, Karolinska Institute, 17177 Stockholm, Sweden
}

\begin{abstract}
Left-right alternation depends on activity in commissural interneurons (CINs) that have axons crossing in the midline. In this study, we investigate the CIN connectivity to local motor neurons using a newly developed preparation of the in vitro neonatal mouse spinal cord that allows us to identify all classes of CINs. Nineteen of 29 short-range CINs with axonal projections $<1.5$ segments (sCINs) directly excited, directly inhibited, or indirectly inhibited contralateral motor neurons in the quiescent spinal cord. Excitation was glutamatergic and inhibition was mixed glycinergic and/or GABAergic. Long-range CINs were also found to have input to local, contralateral motor neurons. Thirteen of 29 descending CINs had similar synaptic connectivity to contralateral motor neurons as the sCINs, including direct excitation and direct and indirect inhibition. Some ( 9 of 23 ) rostrally projecting ascending CINs, and a few ( 2 of 10$)$ CINs with bifurcating axons that both ascend and descend, indirectly inhibited local, contralateral motor neurons. Rhythmic firing during locomotor-like activity was observed in a number of CINs with segmental synaptic effects on contralateral motor neurons. This study outlines the basic connectivity pattern of CINs in the mouse spinal cord on a segmental level. Our study suggests that, based on observed synaptic connectivity, both short- and long-range CINs are likely involved in segmental left-right coordination and that the CIN system is organized into a dual-inhibitory and single-excitatory system. These systems are organized in a way that they could provide appropriate coordination during locomotion.
\end{abstract}

Key words: locomotion; CPG; commissural interneurons; spinal cord; motor neurons; glutamate; GABA; glycine

\section{Introduction}

The neural networks generating rhythmic movements such as swimming and walking are located in the spinal cords of all vertebrates and are called central pattern generators (CPGs). The networks generating swimming in the spinal cord of lamprey and Xenopus tadpole have provided model systems for studying vertebrate CPG function because the basic network structures of these CPGs, as well as many details of the cellular and synaptic mechanisms of CPG function and modulation, have been elucidated (for review, see Roberts et al., 1998; McLean et al., 2000; Buchanan, 2001; Grillner, 2003; Parker, 2006). Commissural interneurons (CINs), whose axons cross the ventral commissure, are a population of neurons common to both lamprey and Xenopus swimming CPGs. They provide reciprocal coordination between left and right sides of the spinal cord and are essential for integrated CPG function. Identification of these neurons and their synaptic connectivity in the lamprey and tadpole swimming CPG was a key initial step in the construction of connectivity maps for these CPGs (Buchanan, 1982; Dale, 1985). Because of this and because CINs are readily identified anatomically and electrophysiologically, CIN connectivity and their control from supraspinal sources has been studied previously quite extensively

\footnotetext{
Received Nov. 23, 2006; revised May 3, 2007; accepted May 4, 2007.

This work was supported by National Institutes of Health Grants R01 NS40795-05 and R01 NS050943. We thank C. E. Restrepo for assistance with confocal imaging and anatomy.

Correspondence should be addressed to Dr. Ole Kiehn at the above address. E-mail: 0.Kiehn@neuro.ki.se. DOI:10.1523/JNEUROSCI.1618-07.2007

Copyright $\odot 2007$ Society for Neuroscience $\quad$ 0270-6474/07/276521-10\$15.00/0
}

in the mammalian lumbar spinal cord. Anatomical studies have described several types of CINs in the ventral, lumbar spinal cord, where the walking CPG is located (Kiehn and Kjaerulff, 1998): (1) long-range CINs that project at least one and a half segments either rostrally [ascending CINs (aCINs)] or caudally [descending CINs (dCINs)], or bifurcate and project in both directions (adCINs), and (2) short-range CINs that project less than one and a half segments [segmental CINs (sCINs)] (Stokke et al., 2002; Matsuyama et al., 2006). Previous functional studies have focused only on the long-range CINs. Descending CINs appear to play a role in coupling flexor-extensor synergies across and along the spinal cord (Butt et al., 2002; Bannatyne et al., 2003; Butt and Kiehn, 2003; Jankowska et al., 2003, 2005a; Krutki et al., 2003; Edgley et al., 2004; Hammar et al., 2004). Ascending CINs are also likely to be involved in locomotor activity based on rhythmicity of firing and neurotransmitter sensitivity (Carlin et al., 2006; Zhong et al., 2006a,b), and some are speculated to play a role in coupling between hindlimbs and forelimbs in quadrupeds (Juvin et al., 2005), or to project to the reticular formation (AntonioGreen et al., 2002; Tran et al., 2004). However, the local synaptic connectivity, which appears to be directly involved in segmental, left-right coordination is largely unknown. Short-range CINs that make up at least $10-15 \%$ of the total population of CINs (Stokke et al., 2002) are likely to be involved in this coordination. They extend axons to contralateral motor neuron pools and the contralateral ventromedial area (Hoover and Durkovic, 1992; Bannatyne et al., 2003; Birinyi et al., 2003; Matsuyama et al., 2006) and most of the last-order premotor CINs are located 
within the same segment (Birinyi et al., 2003). Yet, long-range intersegmental CINs also have local axonal branching (Matsuyama et al., 2004, 2006). In this study, we therefore assessed the local synaptic connectivity of both short- and long-range CINs. Part of this work has been published previously in abstract form (Quinlan and Kiehn, 2005).

\section{Materials and Methods}

Dissection. All procedures were approved by the Swedish Animal Welfare Agency. One hundred and twenty-seven newborn C57BL/6 mice (postnatal day $0-3$ ) were used in this study. They were anesthetized in isoflurane (Forene; Abbott Scandinavia, Solna, Sweden), decapitated, and eviscerated as described previously (Kjaerulff and Kiehn, 1996; Butt et al., 2005). The spinal cord was removed, placed in a recording chamber ventral side up, and perfused with Ringer's solution containing (in $\mathrm{mm}$ ) $111 \mathrm{NaCl}, 3.09 \mathrm{KCl}, 25.0 \mathrm{NaHCO}_{3}, 1.10 \mathrm{KH}_{2} \mathrm{PO}_{4}, 1.26 \mathrm{MgSO}_{4}, 2.52$ $\mathrm{CaCl}_{2}$, and 11.1 glucose (all from Merck, Darmstadt, Germany). Ringer's solution was oxygenated with $95 \% \mathrm{O}_{2}$ and $5 \% \mathrm{CO}_{2}$ and all experiments were performed at room temperature $\left(20-23^{\circ} \mathrm{C}\right)$. The spinal cord was first transversely sectioned at Th10-Th11, then midsagittal lesions were made so that three segments from L1-L2 to L3-L4 were left intact, as shown in Figure $1 A$ in the schematic and in a photo in Figure $1 B$.

Recordings. Both left and right ventral roots (VRs) at either L2 or L3 were suctioned into glass extracellular recording electrodes attached to A1402 $\times 50$ low-noise amplifiers and a Cyberamp 380 (Molecular Devices, Union City, $\mathrm{CA}$ ). Alternating current (AC) channels were recorded with a gain of 5000 and bandpass filtered at 300-1000 Hz. Direct current (DC) channels were recorded at $0.1 \mathrm{~Hz}$, low-pass filtered at $100 \mathrm{~Hz}$ and amplified at least 500 (for more details, see Butt and Kiehn, 2003). Whole-cell patch-clamp recordings were performed blindly from neurons in the ventral L2-L3 segment using borosilicate glass electrodes pulled to a resistance of 5-10 M $\Omega$ (Harvard Apparatus, Kent, UK). Patch electrodes were filled with (in mM) $138 \mathrm{~K}$-gluconate, 10 HEPES, 0.0001 $\mathrm{CaCl}_{2}, 5$ ATP-Mg, and 0.3 GTP-Li (all from Sigma, St. Louis, MO), and in some cases an intracellular tracer (see below). All recordings were performed in current-clamp mode with an Axoclamp 2B (Molecular Devices), and recorded in Axoscope 9.0 at a sampling rate of at $2-5 \mathrm{kHz}$. The location of patched neurons in the ventral cord was estimated by measuring the distance that the patch electrode traveled from the ventral surface using the microdrive counter, and in the transverse plane the distance was measured using the $x-y$ scale on the micromanipulator stand [as described in detail by Butt et al. (2002)].

Stimulation. Commissural interneurons were identified based on the differential ability of three contralateral stimulation electrodes to elicit antidromic spikes in the presence of glutamatergic blockers [either CNQX $(20 \mu \mathrm{M})$ and AP-5 (30 $\mu \mathrm{M})$, or kynurenic acid (0.5 mM) (Jahr and Yoshioka, 1986)] (see Figure 2). Extracellular glass suction electrodes were used to stimulate the rostral (aCINs or adCINs) and caudal hemicords (dCINs or adCINs) and a platinum/iridium concentric bipolar stimulating electrode (12.5 $\mu \mathrm{m}$ diameter; FHC, Bowdoinham, ME) was placed in the ventral midline at L2-L3 to stimulate crossing axons in the midline, as shown in Figures 1 and 2. Axons from CINs cross the midline in the same segment in which the cell body is located with very few exceptions (Eide et al., 1999; Stokke et al., 2002; Matsuyama et al., 2006), so the midline electrode should antidromically activate nearly all CINs located in the intracellular recording segment. All stimulating electrodes were connected to a stimulus isolator (Model M1401 from University of Köln E-Laboratory, Köln, Germany) that was triggered by a Master 8 (A.M.P.I., Jerusalem, Israel) delivering $100 \mu$ s pulses of $10-300 \mu \mathrm{A}$.

Membrane properties. The "resting" membrane potential (RP) was measured from recordings obtained shortly after penetration of the neuron. For neurons displaying spontaneous activity, RP was estimated from the trough of the slow after hyperpolarizations after actions potentials. Input resistance was measured in current clamp from the current-voltage $(I-V)$ relationship of subthreshold current pulses using P-Clamp 9.0. Suites of long (2s) depolarizing and hyperpolarizing current pulses were generated using P-Clamp 9.0. Membrane potentials were not adjusted for the liquid junction potential introduced by the whole-cell configuration (Kiehn et al., 1996; Raastad et al., 1998).

Drugs. D,L-2-amino-5-phosphonovaleric acid, (AP-5; $30 \mu \mathrm{M}$; RBI, Natick, MA) and 6-cyano-7-nitroquinoxaline-2,3-dione (CNQX; $20 \mu \mathrm{M}$; Sigma), or kynurenic acid ( $0.5 \mathrm{~mm}$; Sigma) were used to block fast excitatory synaptic potentials. Glycinergic and GABAergic synaptic potentials were blocked with strychnine $(0.3 \mu \mathrm{M})$ and bicuculline $(3 \mu \mathrm{M})$. Polysynaptic transmission was blocked or reduced by mephenesin (1 mM) (Farkas et al., 1989; Vinay et al., 1995). Locomotor-like activity was induced by perfusion of NMDA $(3.5-10 \mu \mathrm{M})$ and 5-hydroxytryptamine (5-HT; 3.5-15 $\mu \mathrm{M}$ ), occasionally in combination with dopamine (50 $\mu \mathrm{M}$; all from Sigma).

Analysis. Spike-triggered averages were obtained using Datapac software (Run Technologies, Laguna Hills, CA) with files containing at least 300 action potentials, evoked at a rate between 2 and $5 \mathrm{~Hz}$ and usually using $0 \mathrm{mV}$ as the threshold for spike detection similar to what was performed in Butt and Kiehn (2003). Polar plots were performed as described by Butt et al. (2002) to correlate CIN activity with the locomotor rhythm using a custom-designed program written in R (www.rproject.org). Spikes were chosen randomly by $\mathrm{R}$ and then correlated to the phase of locomotor-like activity during which they occurred. Ventral root activity was used as the reference for cellular activity, and was therefore normalized such that the onset of ventral root bursts was defined as $0^{\circ}$ in the circular plot and the subsequent burst onset as $360^{\circ}$, with the time midway through each cycle $180^{\circ}$. Thus, for a cell that fires spikes primarily out of phase with ventral root bursts, the mean point in the circular plot of spikes fired can be expressed as a vector with an angle approximately between $180^{\circ}$ and $360^{\circ}\left(0^{\circ}\right)$. The length of the vector reflects the value of Rayleigh's $R$ (for Rayleigh's test of nonrandom population distribution), so the longer the vector, the greater the significance of the nonrandom distribution. $p$ values were calculated from $R$, and statistical significance was reached when $p \leq 0.05$.

Histology. Intracellular tracers were all diluted in the patch electrode solution and include neurobiotin ( $0.2 \%$; Vector, Burlingame, CA), and Alexa Fluor 488, 568, and 633 (all used at 0.01-0.02\%; Invitrogen, Eugene, OR). After completion of the experiments, preparations were fixed in $4 \%$ paraformaldehyde for $1 \mathrm{~h}$ at $4^{\circ} \mathrm{C}$, washed in PBS, incubated in $20 \%$ sucrose PBS overnight, and frozen in Tissue Tek O.C.T. Compound (Sakura, Zoeterwoude, Netherlands). Preparations were then transversely sliced on a cryostat at $15-50 \mu \mathrm{m}$ thickness, followed by processing for neurobiotin with avidin-conjugated peroxidase when needed, using the Vectastain ABC kit (Vector) and 0.05\% 3,3'diaminobenzidine in PBS. Sections were scanned with an optical thickness of $1 \mu \mathrm{m}$ on an LS 510 Zeiss (Hamburg, Germany) confocal microscope or photographed on a Nikon (Tokyo, Japan) fluorescent microscope. Cells stained for neurobiotin were drawn using a light microscope fitted with a camera lucida and then scanned into CorelDraw.

\section{Results \\ CIN identification}

All neurons that we recorded from were located in laminas VII, VIII, or X, the areas of the rodent spinal cord that contain the neuronal elements necessary for generating locomotor-like activity (Kjaerulff and Kiehn, 1996; Kiehn and Kjaerulff, 1998). As previously, we focused on the upper lumbar spinal cord (L2/L3) (Butt et al., 2002; Butt and Kiehn, 2003). Using consecutive stimulation from three electrodes, we were able to determine (1) whether a cell was commissurally or ipsilaterally projecting and, (2) if the neuron was a CIN, to which of the previously described anatomical classes of commissural interneurons ( $\mathrm{aCIN}, \mathrm{dCIN}$, adCIN, or sCIN) the CIN belonged, as shown in Figures 1 and 2. Glutamatergic blockers (CNQX and AP-5 or kynurenic acid) were always present when detecting antidromic spikes to prevent excitatory synaptic activation of the recorded neuron from the stimulation sites. Although nonglutamatergic putative excitatory interneurons have been reported in the mammalian spinal cord (Huang et al., 2000), no stimulus-evoked excitatory post synaptic 


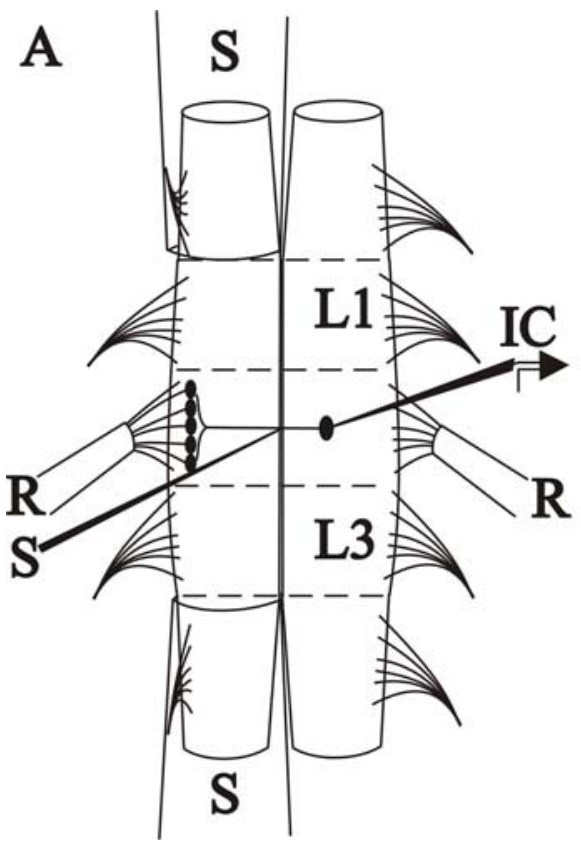

B

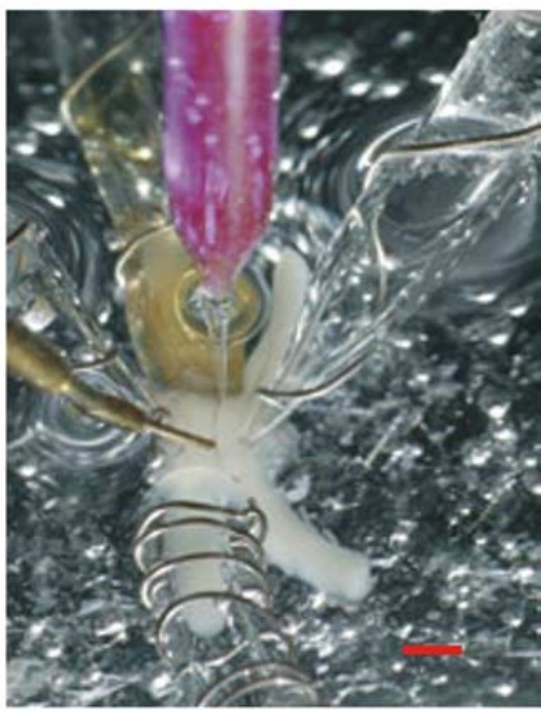

Figure 1. Experimental setup. $A$, Shown schematically, the stimulating electrodes $(S)$ were positioned on rostral and caudal hemicords contralateral from the site of the patch electrode (IC), as well as on the ventral surface of the midline. Extracellular recording electrodes $(R)$ on both left and right ventral roots were used in both $A C$ and $D C$ modes. $B, A$ photograph of the preparation. A total of three intact segments remained in the spinal cords used. Scale bar, (red) $0.5 \mathrm{~mm}$.
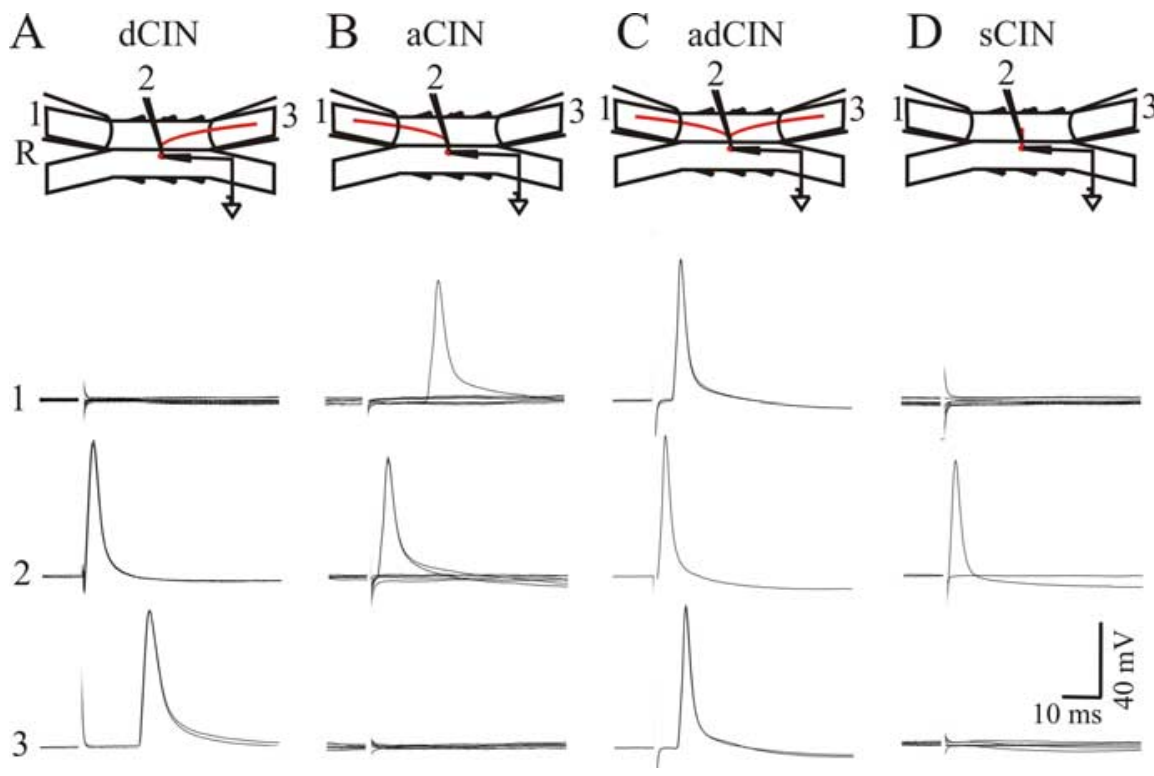

Figure 2. Differential antidromic activation from each of the CIN categories in the presence of glutamate antagonists. $\boldsymbol{A}$, Antidromic spikes in dCINs were elicited from the midline (2) and the caudal, contralateral stimulating electrode (3). $\boldsymbol{B}$, aCINs were identified from spikes with stimulation of rostral hemicord (1) and midline (2). C, Bifurcating adCINs responded to stimulation from all points with an antidromic spike. $\boldsymbol{D}$, scINs spiked only with midline stimulation, indicating that the contralateral projections extended $<1.5$ segments.

potentials were observed in the patched neurons after blockade of AMPA and NMDA receptors with CNQX and AP-5 or kynurenic acid. This is similar to what has been described previously in the rodent spinal cord (Butt et al., 2002; Zhong et al., 2006a,b). With this protocol, antidromic spikes had characteristic sharp onsets and all-or-none behavior like that described for activation of dCINs, adCINs, and aCINs (Butt et al., 2002; Zhong et al., 2006a,b). Stimulation strength, especially at the midline, was carefully adjusted to avoid direct stimulation of the recorded

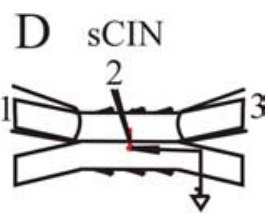

neuron. Using this preparation, 154 neurons were recorded in the ventromedial area. Of those, 106 neurons were electrically defined as commissurally projecting, and 48 were ipsilaterally projecting, including motor neurons. The high yield of CINs compared with the ipsilaterally projecting neurons is a consequence of targeting the ventromedial part of lamina VII and lamina VIII where the concentration of CINs is high (Butt et al., 2002; Butt and Kiehn, 2003; Nissen et al., 2005). Because the midline stimulation electrode by nature was close to the recorded neurons (100-300 $\mu \mathrm{m})$, direct stimulation of neurons was a concern. Such direct stimulation would lead to false classification of neurons as being sCINs when, in reality, they could be ipsilaterally projecting. This was not a concern when classifying dCINs, aCINs, and adCINs because the suction stimulation electrodes on the hemicord were further away from the recording site (Fig. 1) (Butt et al., 2002; Butt and Kiehn, 2003; Zhong et al., 2006a,b). To address this issue, we included, in many cases, intracellular dyes in the patch electrode so that neurons could be identified postexperimentally. In particular, we applied this analysis to those neurons electrically classified as sCINs, but which did not show synaptic connectivity to the contralateral motor neuron pools (for details on synaptic connectivity, see below), and neurons electrically classified as ipsilaterally projecting. Sixteen of the 48 ipsilaterally projecting neurons were filled with intracellular tracers, 11 of which were rescued and had well filled processes. All of these 11 ipsilaterally projecting neurons had only ipsilateral processes, as expected, and in many cases these processes were very close to the midline (Fig. 3A). Seventeen sCINs without contralateral synaptic effects were filled with dye as they were recorded, and of those 17,11 were rescued and had nicely filled processes. Ten of the 11 neurons were confirmed to have processes crossing the midline. Three of these are shown in Figure $3 B-D$. The one remaining neuron did not have any visible contralateral processes and was therefore removed from the totals of CINs. In addition, the sCINs that were recorded, but were not filled (or not well filled) with intracellular tracer and had no contralateral synaptic connectivity were also removed from the totals. In the following analysis, we will therefore focus only on those sCINs that were positively identified in two ways: (1) through both antidromic action potentials and synaptic connectivity with contralateral motor neuron pools (see below), or (2) by both antidromic action potentials and subsequent anatomical confirmation. An example of a sCIN that was synaptically connected to contralateral motor neurons is shown in Figure $3 E$. 
Of the 91 confirmed CINs, 29 were sCINs (which account for $32 \%$ of all CINs recorded in this study), 29 were dCINs (32\% of CINs), 23 were aCINs (25\%), and 10 were adCINs (11\%). These electrophysiologically determined relative numbers are in agreement with previously published numbers from anatomical studies (Stokke et al., 2002) (see Discussion).

\section{Synaptic connectivity of sCINs}

The synaptic connectivity of CINs to motor neurons was determined by recording both ipsilateral and contralateral ventral roots in DC mode from the same segment as the intracellularly recorded neuron. $\mathrm{Re}$ corded cells were depolarized to induce action potentials. In these experiments, 300-1000 action potentials were used for spike-triggered averaging of DC ventral root recordings. This number of spikes was sufficient to determine synaptic effects from interneurons onto motor neurons if present, as described previously in the spinal cords of both chick embryos (Wenner and O'Donovan, 1999) and neonatal rats and mice (Butt and Kiehn, 2003; Butt et al., 2005). Rarely, when CINs were firing spontaneously, periodic depolarizing current was still injected into the cells, inducing more frequent firing and reducing the risk that common synaptic drive was contributing to the averaged response. Additionally, because spontaneous motor bursts are rare in the quiescent cord, the chance of such contamination is probably not pronounced. The DC technique used here is a modification of the sucrose gap technique, which has been used successfully in the adult cat spinal cord to determine synaptic effects from interneurons onto motor neurons (Brink et al., 1983).

There was an observable postsynaptic effect in the contralateral motor neuron pool in response to spike activity in $66 \%$ (19 of 29) of sCINs in the quiescent preparation. A variety of responses both excitatory (Fig. 4A) and inhibitory (Figs. $4 B-C$ ) were observed in motor neurons in response to sCIN firing. Similar to the responses in L4 motor neurons from spiking dCINs in L2 (Butt and Kiehn, 2003), all excitatory responses were blocked by glutamatergic receptor blockers acting on ionotropic glutamate receptors. The synaptic connectivity of sCINs to contralateral motor neurons is summarized in Table 1 and described in detail below.

Excitatory, glutamatergic responses made up 26\% $(n=5)$ of the sCIN input to contralaterally located motor neurons whereas the inhibitory responses accounted for the remaining sCIN input to motor neurons. Of the excitatory responses, two were determined to be direct because they persisted in the presence of the barbiturate mephenesin ( $1 \mathrm{mM})$, which severely reduces the probability of disynaptic and polysynaptic neurotransmission
(Farkas et al., 1989; Vinay et al., 1995; Butt and Kiehn, 2003). The remaining three excitatory responses were not tested in this way. Both direct excitatory responses had a latency of $6 \mathrm{~ms}$ from the peak of the sCIN spike and amplitudes of motor neuronal responses were 16 and $32 \mu \mathrm{V}$. Direct glutamatergic excitation of motor neurons from one sCIN was shown to be mediated by non-NMDA-type glutamate receptors, because it persisted in presence of the NMDA receptor antagonist AP-5 (30 $\mu \mathrm{M})$ (Fig. $4 A$ ), whereas the others were untested for glutamate receptor subtype. The mean amplitude and latency of the excitatory responses were $12.9 \pm 11.6 \mu \mathrm{V}$ and $17 \pm 19 \mathrm{~ms}$, respectively (all values given as $\pm \mathrm{SD}$ throughout).

Fourteen sCINs produced inhibitory responses in contralateral motor neurons. Persistence of an inhibitory response in the presence of glutamatergic antagonists CNQX $(20 \mu \mathrm{M})$ and AP-5 
Table 1. Summary of CIN synaptic connectivity

\begin{tabular}{|c|c|c|c|c|c|c|c|c|}
\hline & \multirow[b]{2}{*}{ Number synaptically connected to contra MNs } & \multicolumn{3}{|c|}{ Excitatory to contralateral MNs } & \multicolumn{4}{|c|}{ Inhibitory to contralateral MNs } \\
\hline & & Incidence & Direct excite & Untested responses & Incidence & Direct inhibit & Indirect inhibit & Untested responses \\
\hline sCIN & 19 of 29 & 5 of 19 & 2 & 3 & 14 of 19 & 4 & 8 & 2 \\
\hline dCIN & 13 of 29 & 2 of 13 & & 2 & 11 of 13 & 4 & 7 & \\
\hline $\mathrm{aClN}$ & 9 of 23 & & & & 9 of 9 & 1 & 6 & 2 \\
\hline $\mathrm{adCIN}$ & 2 of 10 & & & & 2 of 2 & & 2 & \\
\hline
\end{tabular}

MN, Motor neuron.

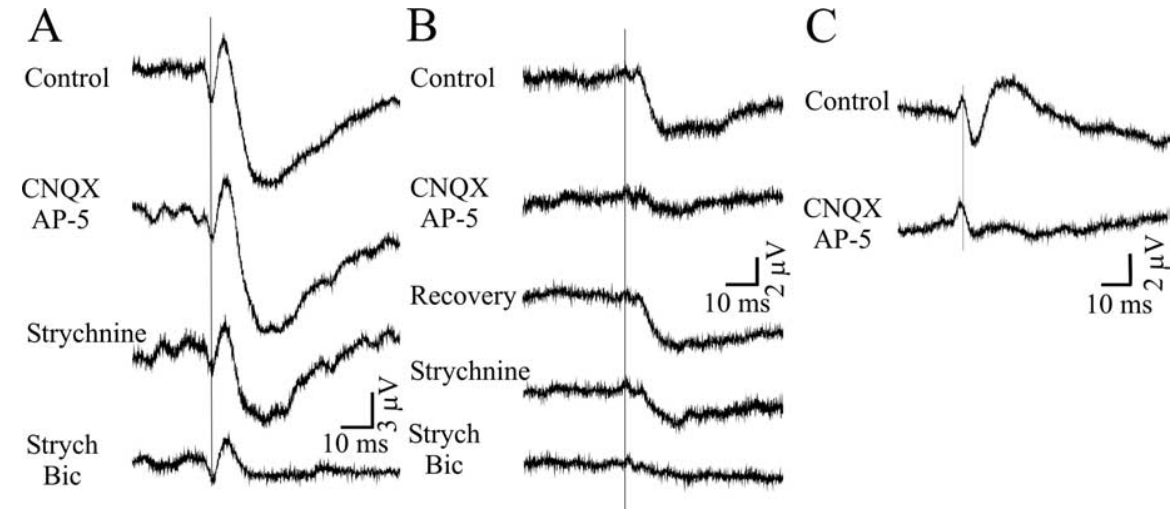

Figure 5. Three examples of synaptic responses in contralateral motor neurons to dCIN firing. Same setup as in Figure 4. $\boldsymbol{A}$, Hyperpolarizing inhibitory response (top) that persists in CNQX and AP5 is partially blocked by strychnine and completely blocked by both strychnine and bicuculline. The short-latency "depolarizing response" that remained after blocking the glutamatergic transmission is part of the stimulus artifact. $\boldsymbol{B}$, Inhibitory response that is attenuated by CNQX and AP5, recovers, and then is partially blocked by strychnine and completely blocked with bicuculline and strychnine. $C$, Excitatory response blocked with CNQX and AP5.

$(30 \mu \mathrm{M})$ was taken as an indication that the CIN-motor neuron connection was direct (Fig. $4 B$ ), because inhibitory responses that are not direct will be mediated by excitatory sCINs synapsing on inhibitory interneurons on the same side as the motor neurons (Butt and Kiehn, 2003) (Fig. 4C). In this way, we found that four sCINs had direct connections to contralateral motor neurons and eight had indirect connections (Table 1), although two were untested. The average latency of direct inhibitory responses was $10 \mathrm{~ms}( \pm 4 \mathrm{~ms})$ with the mean amplitude $9.2 \mu \mathrm{V}( \pm 6.3 \mu \mathrm{V})$. The pharmacology of inhibition was tested in a small number of the inhibitory cells. In three neurons, it appeared to be a mixed glycinergic/GABAergic response, because bicuculline $(3.0 \mu \mathrm{M})$ alone reduced the direct inhibition to an average of $48 \%$ of control (Fig. $4 B)(n=3)$. Inhibition could likewise be reduced to $45 \%$ of control with strychnine $(0.3 \mu \mathrm{M} ; n=1)$, and blocked completely with coapplication of bicuculline and strychnine $(n=$ 1 ). In one neuron, inhibition was blocked completely with bicuculline alone. For nondirect responses, the mean latency was 11 $\mathrm{ms}( \pm 9 \mathrm{~ms})$ and mean amplitude was $6.0 \mu \mathrm{V}( \pm 3.3 \mu \mathrm{V})$.

\section{Local synaptic connectivity of long-range or intersegmental CINs}

In addition to the short-range CINs, long-range or intersegmentally projecting commissural interneurons located in the ventromedial spinal cord were also found to be synaptically connected to motor neurons within the segment of their cell body. Of the long-range CINs, the dCINs had the most abundant $(45 \%$; 13 of 29 dCINs) and the most diverse input to local contralateral motor neurons in the quiescent cord. Similar to sCINs, effects on contralateral motor neurons from dCINs were excitatory and inhibitory, and the inhibitory responses were both direct and indirect. Of the aCINs, 39\% (9 of 23) had synaptic input to local contralat- eral motor neurons. All aCINs recorded were inhibitory to motor neurons. Bifurcating adCINs were the least numerous of the CINs and had the least local output, with 20\% (2 of 10) showing indirect inhibition of motor neurons. These results are summarized in Table 1, and described in more detail below.

\section{Descending commissural interneurons}

Descending commissural interneurons had excitatory and inhibitory, and direct and indirect synaptic input to the contralateral motor neurons within the segment, very similar to the synaptic connectivity of dCINs to caudal, contralateral motor neuron pools described by Butt and Kiehn (2003). Examples of the effects of dCINs on contralateral motor neurons are shown in Figure 5. Of the dCINs that had local synaptic effects, $85 \%$ were inhibitory to motor neurons $(n=11)$, both through direct inhibition $(n=4)$ (Fig. $5 A)$ and indirect inhibition $(n=7)$ (Fig. $5 B$ ). Directness was again determined by the persistence of the response in the presence of glutamatergic blockers (CNQX and AP5). Mean latency was $5 \mathrm{~ms}( \pm 2 \mathrm{~ms})$ and mean amplitude of direct inputs was $3.7 \mu \mathrm{V}( \pm 3.6 \mu \mathrm{V})$. Indirect inhibitory input from dCINs to contralateral motor neurons had an average latency and amplitude of $13 \mathrm{~ms}( \pm 15 \mathrm{~ms})$ and $2.5 \mu \mathrm{V}( \pm 1.6 \mu \mathrm{V})$, respectively. The inhibition from a small number of dCINs was tested pharmacologically and was also found sensitive to blockade with both strychnine and bicuculline, as can be seen in Figure $5 A$, in which a direct inhibitory response was reduced to $60 \%$ of control with strychnine, and completely blocked with coapplication of strychnine and bicuculline. Similarly, indirect inhibition from another dCIN was reduced to $70 \%$ of control with strychnine, and abolished with strychnine and bicuculline, whereas a third indirect inhibitory response was reduced to $40 \%$ of control with strychnine and did not show sensitivity to bicuculline. The nature of this remaining potential is uncertain, but it could be attributable to GABA acting on metabotropic receptors (which we did not test) or release of an inhibitory transmitter unknown to us. Excitatory input to the local contralateral motor neurons comprised $15 \%$ of the dCIN input $(n=2)$ (Fig. $5 C$ ). The latencies and amplitudes of the excitatory responses were eight and $10 \mathrm{~ms}$ and 2.0 and $2.3 \mu \mathrm{V}$, and, unfortunately, neither was tested with mephenesin.

\section{Ascending commissural interneurons}

All nine of the 23 aCINs that had local input to contralateral motor neurons produced inhibitory responses. The inhibition was mainly indirect; in six of the seven cases tested it was blocked by glutamatergic blockers, indicating a dependence on excitatory neurotransmission. The average latency and amplitude of the 
indirect inhibition was $12 \mathrm{~ms}$ ( $\pm 9 \mathrm{~ms}$ ) and $1.8 \mu \mathrm{V}( \pm 0.4 \mu \mathrm{V})$, respectively. In one case, a small $(0.2 \mu \mathrm{V})$ inhibition remained in the presence of glutamatergic blockers, which was then blocked with strychnine (latency, $11 \mathrm{~ms}$ ) (see Discussion).

\section{Bifurcating commissural interneurons}

The adCINs had the least synaptic input to local motor neurons. Only two of 10 produced inhibition of contralateral motor neurons, with amplitudes and latencies of 0.8 and $5.4 \mu \mathrm{V}$ and 8 and $10 \mathrm{~ms}$, respectively. In both cases, the inhibition was blocked with glutamatergic antagonists, indicating that the effects were indirect. One response was also tested with the GABAergic antagonist bicuculline and persisted, suggesting that the response was mediated by glycine.

\section{Ipsilateral effects}

Because ipsilateral axonal branching of CINs has been described in both newborn (Scheibel and Scheibel, 1969) and adult (Matsuyama et al., 2006) mammals, we checked all CINs for possible effects on ipsilateral, segmental motor neurons. In a few cases, there were synaptic effects from CINs onto these motor neurons, detected from the DC ventral root recording. In total, 5 of 91, or $5 \%$ of all CINs had some input to ipsilateral motor neurons. These effects originated from one sCIN, three dCINs, and one aCIN. In three cases, the ipsilateral motor neurons were inhibited (two indirectly, one untested), and in two cases the effects were excitatory (both untested). Two of these five neurons (one sCIN, one dCIN) also had synaptic effects on contralateral motor neurons in the same segment. The ipsilateral effects of both neurons were inhibitory, both ipsilaterally and contralaterally, which means that these neurons were excitatory CINs indirectly inhibiting the motor neurons on either side of the cord. Mean ipsilateral amplitude and latency for all five neurons were $6.3 \mu \mathrm{V}( \pm 4.9$ $\mu \mathrm{V})$ and $18 \mathrm{~ms}( \pm 15 \mathrm{~ms})$, respectively. The latencies for those cells with bilateral effects were in the same range on both sides.

\section{Activity of the CINs during locomotor-like activity}

As an additional functional characterization of the recorded CINs, we wanted to record their activity during drug-induced locomotor-like activity (5-HT and NMDA or 5-HT, NMDA, and dopamine). Unfortunately, in the reduced preparation necessary to unambiguously identify short-range projecting CINs, the locomotor-like activity was generally weak, because of the reduction in the size of the locomotor network left intact. Any preparations that did not produce regular, alternating activity in left and right ventral roots were eliminated from the analysis. Neurons referred to as inhibitory or excitatory to motor neurons in this section were determined as such based on their synaptic effect on motor neurons in the quiescent cord. It should be noted, however, that state-dependent changes in synaptic connectivity could be occurring during locomotor activity, potentially changing the nature of the synaptic effects of CINs onto motor neurons, as described previously (Jankowska et al., 1967; Gossard et al., 1994; McCrea, 2001; Butt and Kiehn, 2003; Angel et al., 2005).

Of the sCINs recorded in those preparations capable of producing locomotor-like activity, 5 of 10 sCINs displayed significantly rhythmic activity, whereas the remaining cells were only weakly related to the ventral root bursting. Of these five rhythmic
sCINs, two fired action potentials out of phase with the contralateral VR bursts (Fig. 6), both of which were found to inhibit contralateral motor neurons in the quiescent cord. The other three sCINs were active in phase with the contralateral VR bursts. One of the three was exciting contralateral motor neurons and the other two sCINs had no detectable synaptic output to local, contralateral motor neurons in the quiescent cord, but both were confirmed to be sCINs anatomically. An example of a rhythmically firing sCINs is shown in Figure 6, with raw traces of sCIN and contralateral ventral root activity shown in Figure $6 \mathrm{~A}$ while the spikes fired by the cell are correlated to the locomotor cycle in a circular plot in Figure $6 \mathrm{~B}$. The portion of the plot from 0 to $106^{\circ}$ represents the mean burst duration of the contralateral ventral root; so the activity of this cell corresponds to the period when the contralateral root is silent. The preferred firing phase of this cell was $241^{\circ}$, as shown in the circular plot $(p<0.001)$. The other inhibitory sCIN was also most active during the silent period of the contralateral ventral root $\left(199^{\circ} ; p=0.02\right)$. The preferred phase of firing of the excitatory sCIN was at the end of the contralateral ventral root burst $\left(151^{\circ} ; p<0.01\right)$, suggesting a direct crossed activation of contralateral motor neurons. The two sCINs with no synaptic effect on local motor neurons were most active during the peak burst activity of the contralateral ventral root (64 and $70^{\circ}$; both $\left.p<0.01\right)$.

The relatively low number of rhythmically active sCINs (50\%) that we report in the present study could be a reflection of the reduced locomotor capacity of this short preparation. In a more fully intact, rhythmically active rat spinal cord, rhythmicity is found in $\sim 70 \%$ of L2 neurons (Tresch and Kiehn, 1999) including a majority of ascending, descending and long-range projecting CINs [aCINs and projecting CINs, cat (Huang et al., 2000; Matsuyama et al., 2004), cat; dCINs and aCINs, rodent (Butt and Kiehn, 2003; Zhong et al., 2006a,b)]. Yet, the other groups of CINs were also found to be less rhythmically active in this study. Thus, only $25 \%$ ( 2 of 8 ) aCINs and no (0 of 3 ) dCINs were found to be significantly rhythmic in the present study. One of the rhythmically active aCINs recorded in this study was inhibitory to contralateral motor neurons in the quiescent cord and fired action potentials at the end of contralateral ventral root bursts (preferred phase of firing $156^{\circ} ; p=0.02$ ). The other aCIN had a preferred phase of firing at $234^{\circ}(p<0.001)$, but had no input to local motor neurons. None of the adCINs recorded in this study were rhythmically active during locomotor-like activity ( 0 of 3 adCINs were significantly rhythmic). Thus, both the locomotorrelated firing patterns and the synaptic connectivity of the shortrange (and possibly the projecting) CINs suggest that they take an active role in segregating left-right locomotor-related activity. 

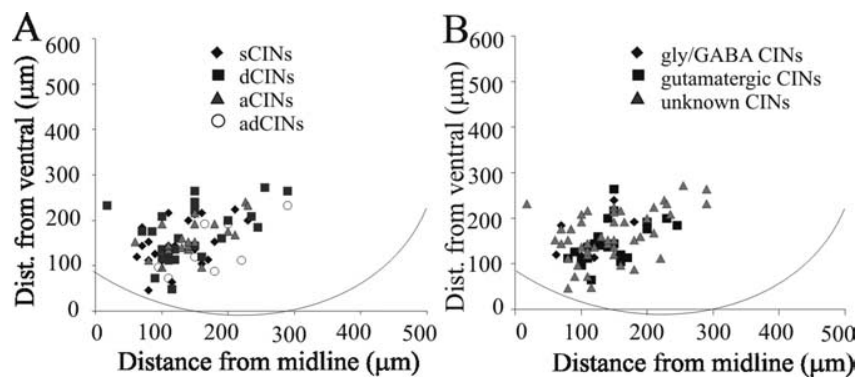

Figure 7. Locations of the CINs recorded in the ventromedial spinal cord. All neurons were patched in lumbar segments $\mathrm{L} 2$ and $\mathrm{L} 3$. $\boldsymbol{A}$, The location of recorded CINs grouped by axonal projection. $\boldsymbol{B}$, Location of CINs grouped by neurotransmitter phenotype. CINs were distributed throughout the ventromedial area without any apparent grouping by neurotransmitter phenotype or axonal projection.

\section{Location CINs recorded}

The CINs recorded in this study were all located in the ventromedial area of L2 and L3, and the approximate locations of 75 CINs of all types are plotted in Figure 7A. The distribution of CIN classes in the transverse plane of the newborn rodent are in accordance with previous rodent studies (Eide et al., 1999; Stokke et al., 2002; Birinyi et al., 2003; Nissen et al., 2005), with broad overlap between the ventral sCINs, dCINs, aCINs, and adCINs. There was also no clear distribution of CINs in the transverse plane when they were plotted according to their transmitter phenotype (Fig. 7B).

\section{Membrane properties of CINs}

Some basic membrane properties of different categories of CINs were tested in this study, including mean resting membrane potential and input resistance of all CINs, which were $-50 \mathrm{mV}( \pm 6$ $\mathrm{mV}$ and $n=57)$ and $704 \mathrm{M} \Omega( \pm 338 \mathrm{M} \Omega$ and $n=54)$, respectively. These values were not significantly different between the categories of CINs, as listed in Table 2. Moreover, some CINs in all categories displayed both hyperpolarizing sag [a sign of hyperpolarization-activated cation currents $\left.\left(I_{\mathrm{h}}\right)\right]$ and postinhibitory rebound [PIR; a sign of $I_{\mathrm{h}}$ and/or transient calcium currents $\left.\left(I_{\mathrm{t}}\right)\right]$ in response to a hyperpolarizing current injection, whereas some CINs showed only PIR, as shown in two sCINs in Figure 8. These responses seem to be distributed equally among the commissural interneurons (Table 2).

\section{Discussion}

Our experiments show that sCINs have excitatory glutamatergic and inhibitory glycinergic/GABAergic inputs to contralateral motor neurons. In addition, long range intersegmental CINs were found to have excitatory and inhibitory synaptic inputs to local motor neurons. The general layout of these connections is discussed below.

\section{Methodological considerations}

Neurons were identified by the pattern of antidromic action potentials elicited from the ventral midline surface and the contralateral hemicord rostral and caudal to the recording site by one and a half segments. Neurons found by this procedure could potentially have been misclassified as ipsilaterally projecting neurons because of direct stimulation. Yet, to avoid any potential miscategorization, all sCINs that did not have contralateral effects and that could not be anatomically confirmed were not included in the analysis.

The use of spike-triggered averaging of DC recordings to re- veal synaptic connectivity has been thoroughly evaluated in a previous study (Butt and Kiehn, 2003). One caveat to the technique is the possibility that signal could be contaminated by inputs to motor neurons from common excitation pathways that contribute excitation to CINs as well (Rudomin et al., 1987). This is especially problematic during network activity. Periods of large spontaneous burst activity were therefore discarded from the analysis. As previously reported (Butt and Kiehn, 2003), we were able to detect excitation as depolarization and inhibition as hyperpolarization. The synaptic nature of these responses was confirmed pharmacologically in most cases. Previous findings in the hippocampus and neocortex indicate that a transient oxytocinassociated downregulation of NKCC1 (sodium potassium chloride cotransporter) activity perinatally shifts the chloride reversal potential to a more hyperpolarized level, making inhibitory potentials hyperpolarizing at rest around the time of birth (Tyzio et al., 2006). This effect is potentially a contributing factor in our experiments, in which most $(72 \%)$ neurons were patched in spinal cords from mice within the first day of birth.

Another caveat to consider in interpreting our findings is that, because categorization further subdivides the anatomically grouped CINs into groups based on synaptic connectivity, we end up with relatively low $n$ numbers in each category, especially for the pharmacological findings. We cannot therefore exclude that this listing of CIN synaptic connectivity and pharmacology is potentially incomplete. We believe, however, that the broad categories we found provide a representative sample of the existing synaptic connectivity.

\section{Pharmacology of CINs}

\section{Excitatory CINs}

The majority of premotor and pre-premotor CINs recorded in this study were glutamatergic. Excitatory glutamatergic CINs were found to have a dual function: (1) direct (and likely indirect) excitation of contralateral motor neurons, and (2) inhibition of contralateral motor neurons via excitation of local inhibitory interneurons.

Monosynaptic excitation of contralateral motor neurons by CINs was shown here directly for the first time, although glutamatergic CINs excited by reticulospinal neurons and projecting directly to contralateral motor neurons have been shown indirectly in adult cats (Bannatyne et al., 2003; Jankowska et al., 2003, 2005b; Hammar et al., 2004; Matsuyama and Jankowska, 2004). Glutamatergic dCINs have also been shown to directly excite caudal, contralateral motor neurons in neonatal rats (Butt and Kiehn, 2003). In this study, we now show that both sCINs and dCINs contribute excitation to local, contralateral motor neurons.

Most of the glutamatergic CINs are actually inhibiting contralateral motor neurons via local, ipsilateral, inhibitory interneurons. Based on previous work, these inhibitory interneurons are expected to include (but are not limited to) Renshaw cells and Ia inhibitory interneurons (Fig. 9). Excitation from CINs onto Ia inhibitory interneurons has been shown in the adult cat spinal cord (Jankowska et al., 2005c) and Renshaw cells have been shown to receive both excitatory and inhibitory input from commissural axons in the neonatal mouse spinal cord (Nishimaru et al., 2006). The pharmacology was tested in a few cases of indirect inhibition and results were mixed with both glycinergic and GABAergic contribution. One aCIN weakly inhibited contralateral motor neurons through an ionotropic glutamate-receptor independent mechanism, suggesting that CINs can act via 
metabotropic glutamate receptors or that they contain other excitatory transmitters (e.g., acetylcholine) (Huang et al., 2000).

Inhibitory CINs

sCINs and dCINs had direct inhibitory inputs to motor neurons. The inhibitory pharmacology of these connections was tested in a subsample of CINs and shown to be glycinergic/GABAergic. If these findings are extrapolated, $20 \%$ of sCINs and $30 \%$ of dCINs with synaptic connectivity to motor neurons are glycinergic/ GABAergic in the mouse. Although there appears to be a strong GABAergic commissural presence in the embryonic rodent spinal cord based on both anatomical and electrophysiological data (Ma et al., 1992a,b; Nishimaru and Kudo, 2000; Nakayama et al., 2002; Tran et al., 2003, 2004), neither the CINs in adult cat (Bannatyne et al., 2003; Matsuyama and Jankowska, 2004) nor the dCINs in newborn rat (Butt and Kiehn, 2003) appear to retain GABA as a neurotransmitter. It is possible that GABAergic CINs are a transitional phenomenon. However, the contributions of GABA to locomotor activity in rodents might have been overlooked. In fact, studies using concentrations and antagonists that selectively block GABA and not glycine have shown an involvement in left-right coordination of locomotor activity in the spinal cord of both mice and rats (Pflieger et al., 2002; Hinckley et al., 2005).

\section{Local input of long-range projecting CINs}

Here, we show that $40 \%$ of long-range projecting CINs also have local synaptic contacts. It has been documented that CINs have axon collaterals that remain within the same segment, and some CINs have numerous axonal branches along the entire length of their axonal trajectory (Eide et al., 1999; Bannatyne et al., 2003; Matsuyama et al., 2004, 2006). However, until now, these connections have not been shown to be functional. It is therefore likely that CINs act in concert to bring about the proper coordination of the locomotor CPG on the left and right sides of the cord.

\section{Membrane properties of CINs}

Some membrane properties of CINs were measured in this study. The present results suggest that the membrane properties were not distinct for any anatomical category. This is in line with previous studies of aCINs, dCINs, and adCINs, which have shown that resting membrane potentials, input resistances, and complements of sag and PIR are similar in these categories of CINs (Butt et al., 2002; Zhong et al., 2006a,b). These properties are furthermore shared by other putative CPG interneurons in the rodent (Wilson et al., 2005; Hinckley and Ziskind-Conhaim, 2006).

\section{Overall functional organization}

From our study, it appears that the structure of the network controlling motor neurons at the segmental level has two functional pathways: inhibitory and excitatory. The inhibitory CIN system mediates its effect via (1) indirect inhibition mediated by

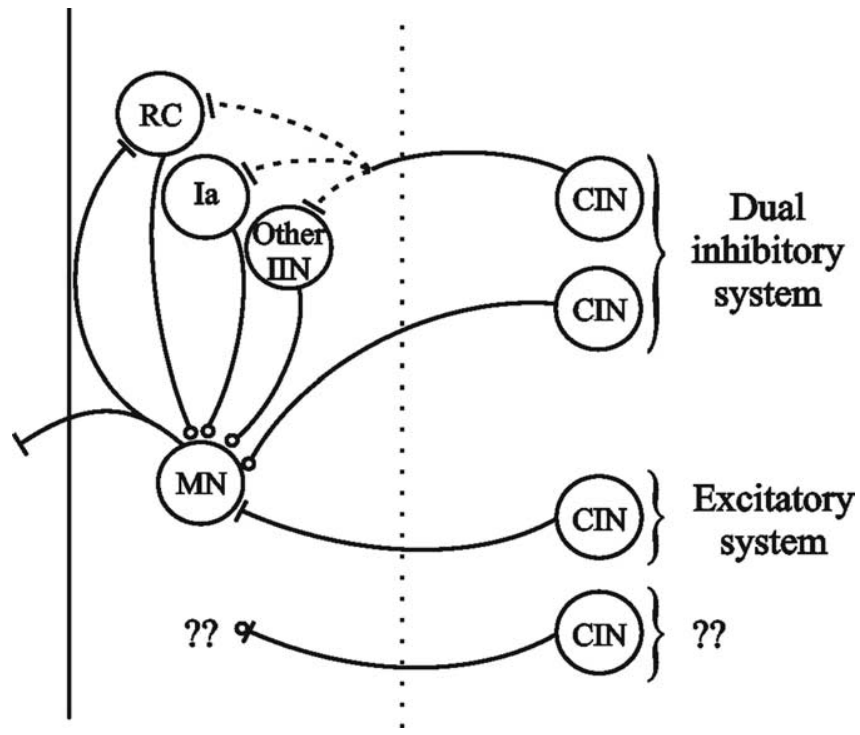

Figure 9. Summary diagram of local CIN connections. Inhibitory synapses are represented by closed circles whereas excitatory synapses are represented by flat lines. MN, Motor neuron; RC, Renshaw cells; IIN, ipsilateral inhibitory interneuron. The figure is modified from Kiehn (2006)

glutamatergic CINs and local, ipsilaterally projecting, inhibitory interneurons, and (2) monosynaptic inhibition, which appears to be mediated by mixed glycinergic/GABAergic CINs at this age (Fig. 9). The local inhibitory neurons can be either Ia interneurons, Renshaw cells, or another unknown class of inhibitory neurons (Jankowska et al., 2005c; Nishimaru et al., 2006). We propose that this dual system of inhibition is active during 
alternating rhythmic activity; although, because of the poor locomotor-like activity found in this preparation, only three inhibitory CINs were confirmed rhythmically active in the appropriate phase. The intrasegmental excitatory CIN system is composed of glutamatergic CINs that are able to excite motor neurons directly. The excitatory CIN system could serve two roles: one is to be active during alternation so that excitation is transmitted across the cord to enhance contralateral motor activity. In this case, extensor activity could be linked together with flexor activity on the contralateral side in the same segment. Preliminary evidence for this was found here. Another possible functional role of the segmental excitatory CIN system is that it is active during bilaterally synchronous activity, binding left-right segmental activity (e.g., the coactivation of flexors as seen during hopping). This activity was not expected to be active under circumstances created in this study, where locomotor-like activity was alternating when successfully induced.

Ipsilateral connections were considerably less frequent than contralateral connections and only $2 \%$ of CINs had connections on both sides. The functional role of CIN connections to ipsilateral motor neurons is not clear. Perhaps they are contributing to organizing muscle synergies across the cord.

The connectivity found in the quiescent preparations might not represent the full repertoire of connections seen during locomotor-like activity (Jankowska et al., 1967; Gossard et al., 1994; McCrea, 2001; Angel et al., 2005). In a previous study of dCIN connections, state dependent changes were seen in a small population of indirectly coupled dCINs (Butt and Kiehn, 2003). It is possible that not only could connections change their excitatory or inhibitory nature, but also that some connections could drop out or appear in a state dependent manner.

Although the segmental CIN synaptic connectivity to motor neurons will assist in determining the motor neuronal pattern of activity, these connections alone are unlikely to be responsible for the left-right coordination during locomotor activity. Rather, CINs must also connect to neurons in CPG-neurons to effect the alternation. It is therefore possible that some of the CINs that did not connect to motor neurons may be connecting to contralateral CPG neurons including other contralateral CINs, as has been revealed anatomically (Birinyi et al., 2003, Matsuyama et al., 2006). A major aim of future work is to determine the connectivity of these circuitries.

\section{References}

Angel MJ, Jankowska E, McCrea DA (2005) Candidate interneurons mediating group I disynaptic EPSPs in extensor motoneurones during fictive locomotion in the cat. J Physiol (Lond) 563:597-610.

Antonio-Green DM, Cheng J, Magnuson DS (2002) Neurons labeled from locomotor-related ventrolateral funiculus stimulus sites in the neonatal rat spinal cord. J Comp Neurol 442:226-238.

Bannatyne BA, Edgley SA, Hammar I, Jankowska E, Maxwell DJ (2003) Networks of inhibitory and excitatory commissural interneurons mediating crossed reticulospinal actions. Eur J Neurosci 18:2273-2284.

Birinyi A, Viszokay K, Weber I, Kiehn O, Antal M (2003) Synaptic targets of commissural interneurons in the lumbar spinal cord of neonatal rats. J Comp Neurol 461:429-440.

Brink E, Jankowska E, McCrea DA, Skoog B (1983) Inhibitory interactions between interneurones in reflex pathways from group $\mathrm{Ia}$ and group $\mathrm{Ib}$ afferents in the cat. J Physiol (Lond) 343:361-373.

Buchanan JT (1982) Identification of interneurons with contralateral, caudal axons in the lamprey spinal cord: synaptic interactions and morphology. J Neurophys 47:961-975.

Buchanan JT (2001) Contributions of identifiable neurons and neuron classes to lamprey vertebrate neurobiology. Prog Neurbiol 63:441-466.

Butt SJ, Kiehn O (2003) Functional identification of interneurons responsi- ble for left-right coordination of hindlimbs in mammals. Neuron 38:953-963.

Butt SJ, Harris-Warrick RM, Kiehn O (2002) Firing properties of identified interneuron populations in the mammalian hindlimb central pattern generator. J Neurosci 15:9961-9971.

Butt SJ, Lundfald L, Kiehn O (2005) EphA4 defines a class of excitatory locomotor-related interneurons. Proc Natl Acad Sci USA 102:14098-14103.

Carlin KP, Dai Y, Jordan LM (2006) Cholinergic and serotonergic excitation of ascending commissural neurons in the thoraco-lumbar spinal cord of the neonatal mouse. J Neurophyiol 95:1278-1284.

Dale N (1985) Reciprocal inhibitory interneurones in the Xenopus embryo spinal cord. J Physiol (Lond) 363:61-70.

Edgley SA, Jankowska E, Hammar I (2004) Ipsilateral actions of feline corticospinal tract neurons on limb motoneurons. J Neurosci 24:7804-7813.

Eide AL, Glover J, Kjaerulff O, Kiehn O (1999) Characterization of commissural interneurons in the lumbar region of the neonatal rat. J Comp Neurol 403:332-345.

Farkas S, Tarnawa I, Berzsenyi P (1989) Effects of some centrally acting muscle relaxants on spinal root potentials: a comparative study. Neuropharmacology 28:161-173.

Gossard JP, Brownstone RM, Barajon I, Hultborn H (1994) Transmission in a locomotor-related group $\mathrm{Ib}$ pathway from hindlimb extensor muscles in the cat. Exp Brain Res 98:213-228.

Grillner S (2003) The motor infrastructure: from ion channels to neuronal networks. Nat Rev 4:573-586.

Hammar I, Bannatyne BA, Maxwell DJ, Edgley SA, Jankowska E (2004) The actions of monoamines and distribution of noradrenergic and serotoninergic contacts on different subpopulations of commissural interneurons in the cat spinal cord. Eur J Neurosci 19:1305-1316.

Hinckley C, Seebach B, Ziskind-Conhaim L (2005) Distinct roles of glycinergic and GABAergic inhibition in coordinating locomotor-like rhythms in the neonatal mouse spinal cord. Neuroscience 131:745-758.

Hinckley CA, Ziskind-Conhaim L (2006) Electrical coupling between locomotor-related excitatory interneurons in the mammalian spinal cord. J Neurosci 26:8477-8483.

Hoover JE, Durkovic RG (1992) Retrograde labeling of lumbosacral interneurons following injections of red and green fluorescent microspheres into hindlimb motor nuclei of the cat. Somatosens Mot Res 9:211-226.

Huang A, Noga BR, Carr PA, Fedirchuk B, Jordan LM (2000) Spinal cholinergic neurons activated during locomotion: localization and electrophysiological characterization. J Neurophysiol 83:3537-3547.

Jahr CE, Yoshioka K (1986) Ia afferent excitation of motoneurones in the in vitro new-born rat spinal cord is selectively antagonized by kynurenate. J Physiol (Lond) 370:515-530.

Jankowska E, Jukes MG, Lund S, Lundberg A (1967) The effect of DOPA on the spinal cord. 5. Reciprocal organization of pathways transmitting excitatory action to alpha motoneurones of flexors and extensors. Acta Physiol Scand 70:369-388.

Jankowska E, Hammar I, Slawinska U, Maleszak K, Edgley SA (2003) Neuronal basis of crossed actions from the reticular formation on feline hindlimb motoneurons. J Neurosci 23:1867-1878.

Jankowska E, Edgley SA, Krutki P, Hammar I (2005a) Functional differentiation and organization of feline midlumbar commissural interneurons. J Physiol (Lond) 565:645-658.

Jankowska E, Cabaj A, Pettersson LG (2005b) How to enhance ipsilateral actions of pyramidal tract neurons. J Neurosci 25:7401-7405.

Jankowska E, Krutki P, Matsuyama K (2005c) Relative contribution of Ia inhibitory interneurons to inhibition of feline contralateral motoneurones evoked via commissural interneurons. J Physiol (Lond) 568:617-628.

Juvin L, Simmers J, Morin D (2005) Propriospinal circuitry underlying interlimb coordination in mammalian quadrupedal locomotion. J Neurosci 25:6025-6035.

Kiehn O (2006) Locomotor circuits in the mammalian spinal cord. Annu Rev Neurosci 29:279-306.

Kiehn O, Kjaerulff O (1998) Distribution of central pattern generators for rhythmic motor outputs in the spinal cord of limbed vertebrates. Ann NY Acad Sci 860:110-129.

Kiehn O, Johnson BR, Raastad M (1996) Plateau properties in mammalian spinal interneurons during transmitter-induced locomotor activity. Neuroscience 75:263-273. 
Kjaerulff O, Kiehn O (1996) Distribution of networks generating and coordinating locomotor activity in the neonatal rat spinal cord: a lesion study. J Neurosci 16:5777-5794.

Krutki P, Jankowska E, Edgley SA (2003) Are crossed actions of reticulospinal and vestibulospinal neurons on feline motoneurons mediated by the same or separate commissural neurons? J Neurosci 23:8041-8050.

Ma W, Behar T, Maric D, Maric I, Barker JL (1992a) Neuroepithelial cells in the rat spinal cord express glutamate decarboxylase immunoreactivity in vivo and in vitro. J Comp Neurol 325:257-270.

Ma W, Behar T, Barker JL (1992b) Transient expression of GABA immunoreactivity in the developing rat spinal cord. J Comp Neurol 325:271-290.

Matsuyama K, Jankowska E (2004) Coupling between feline cerebellum (fastigial neurons) and motoneurons innervating hindlimb muscles. J Neurophysiol 91:1183-1192.

Matsuyama K, Nakajima K, Mori F, Aoki M, Mori S (2004) Lumbar commissural interneurons with reticulospinal inputs in the cat: morphology and discharge patterns during fictive locomotion. J Comp Neurol 474:546-561.

Matsuyama K, Kobayashi S, Aoki M (2006) Projection patterns of lamina VIII commissural neurons in the lumbar spinal cord of the adult cat: an anterograde neural tracing study. Neuroscience 140:203-218.

McCrea DA (2001) Spinal circuitry of sensorimotor control of locomotion. J Physiol (Lond) 533:41-50.

McLean DA, Merrywest SD, Sillar KT (2000) The development of neuromodulatory systems and the maturation of motor patterns in amphibian tadpoles. Brain Res Bull 53:595-603.

Nakayama K, Nishimaru H, Kudo N (2002) Basis of changes in left-right coordination of rhythmic motor activity during development in the rat spinal cord. J Neurosci 22:10388-10398.

Nishimaru H, Kudo N (2000) Formation of the central pattern generator for locomotion in the rat and mouse. Brain Res Bull 53:661-669.

Nishimaru H, Restrepo CE, Kiehn O (2006) Activity of Renshaw cells during locomotor-like rhythmic activity in the isolated spinal cord of neonatal mice. J Neurosci 26:5320-5328.

Nissen U, Mochida H, Glover JC (2005) Development of projection-specific interneurons and projection neurons in the embryonic mouse and rat spinal cord. J Comp Neurol 483:30-47.

Parker D (2006) Complexities and uncertainties of neuronal network function. Philos Trans R Soc Lond B Biol Sci 361:81-99.

Pflieger JF, Clarac F, Vinay L (2002) Picrotoxin and bicuculline have different effects on lumbar spinal networks and motoneurons in the neonatal rat. Brain Res 935:81-86.

Quinlan KA, Kiehn O (2005) Synaptic effects of intrasegmental commissural interneurons in the mouse spinal cord. Soc Neurosci Abstr 31:516.1.
Raastad M, Enriquez-Denton M, Kiehn O (1998) Synaptic signaling in an active central network only moderately changes passive membrane properties. Proc Natl Acad Sci USA 95:10251-10256.

Roberts A, Soffe SR, Wolf ES, Yoshida M, Zhao FY (1998) Central circuits controlling locomotion in young frog tadpoles. Ann NY Acad Sci 860:19-34.

Rudomin P, Solodkin M, Jiménez I (1987) Synaptic potentials of primary afferent fibers and motoneurons evoked by single intermediate nucleus interneurons in the cat spinal cord. J Neurophysiol 57:1288-1313.

Scheibel ME, Scheibel AB (1969) A structural analysis of spinal interneurons and Renshaw cells. UCLA Forum Med Sci 11:159-208.

Stokke MF, Nissen U, Glover JC, Kiehn O (2002) Projection patterns of commissural interneurons in the lumbar spinal cord of the neonatal rat. J Comp Neurol 446:349-359.

Tran TS, Alijani A, Phelps PE (2003) Unique developmental patterns of GABAergic neurons in rat spinal cord. J Comp Neurol 456:112-126.

Tran TS, Cohen-Cory S, Phelps PE (2004) Embryonic GABAergic spinal commissural neurons project rostrally to mesencephalic targets. J Comp Neurol 475:327-339.

Tresch MC, Kiehn O (1999) Coding of locomotor phase in populations of neurons in rostral and caudal segments of the neonatal rat lumbar spinal cord. J Neurophysiol 82:3563-3574.

Tyzio R, Cossart R, Khalilov I, Minlebaev M, Hübner CA, Represa A, Ben-Ari Y, Khazipov R (2006) Maternal oxytocin triggers a transient inhibitory switch in GABA signalling in the fetal brain during delivery. Science 314:1788-1792.

Vinay L, Cazalets JR, Clarac F (1995) Evidence for a functional polysynaptic pathway from trigeminal afferents to lumbar motoneurons in the neonatal rat. Eur J Neurosci 7:143-151.

Wenner P, O'Donovan MJ (1999) Identification of an interneuronal population that mediates recurrent inhibition of motoneurons in the developing chick spinal cord. J Neurosci 19:7557-7567.

Wilson JM, Hartley R, Maxwell DJ, Todd AJ, Lieberam I, Kaltschmidt JA, Yoshida Y, Jessell TM, Brownstone RM (2005) Conditional rhythmicity of ventral spinal interneurons defined by expression of the $\mathrm{Hb} 9$ homeodomain protein. J Neurosci 25:5710-5719.

Zhong G, Diaz-Rios M, Harris-Warrick RM (2006a) Serotonin modulates the properties of ascending commissural interneurons in the neonatal mouse spinal cord. J Neurophysiol 95:1545-1555.

Zhong G, Diaz-Rios M, Harris-Warrick RM (2006b) Intrinsic and functional differences among commissural interneurons during fictive locomotion and serotonergic modulation in the neonatal mouse. J Neurosci 26:6509-6517. 